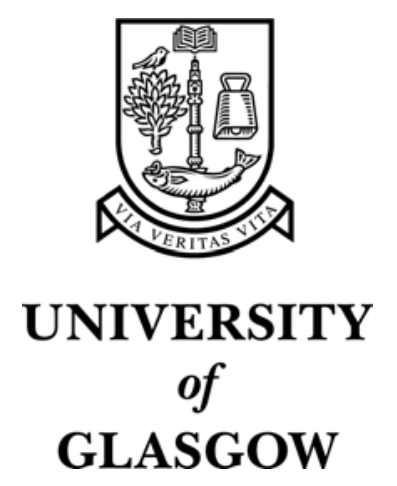

Tsirimonaki, E. and Ullah, R and Marchetti, B. and Ashrafi, G.H. and McGarry, L. and Ozanne, B. and Campo, M.S. (2006) Similarities and differences between the E5 oncoproteins of bovine papillomaviruses type 1 and type 4: Cytoskeleton, motility and invasiveness in E5-transformed bovine and mouse cells. Virus research 115(2):pp. 158-168.

http://eprints.gla.ac.uk/3095/ 


\title{
Similarities and differences between the E5 oncoproteins of bovine papillomaviruses type 1 and type 4 : cytoskeleton, motility and invasiveness in E5-transformed bovine and mouse cells
}

\author{
Emmanuella Tsirimonaki ${ }^{1}$, Robina Ullah ${ }^{1}$, Barbara Marchetti ${ }^{1}$, \\ G Hossein Ashrafi ${ }^{1}$, Lynn McGarry ${ }^{2}$, Brad Ozanne ${ }^{2}$, M Saveria Campo ${ }^{{ }^{*}}$ \\ ${ }^{1}$ Institute of Comparative Medicine, University of Glasgow \\ ${ }^{2}$ Beatson Institute for Cancer Research \\ Glasgow, Scotland
}

Short title: Bovine papillomavirus E5 cell transformation

\author{
Abstract: 181 words \\ Text (excluding references and Figure Legends): 3876 words \\ Number of Figures: 6 \\ Number of tables: 1 \\ Correspondence to Prof MS Campo, Institute of Comparative Medicine, Glasgow \\ University, Garscube Estate, Glasgow G61 1QH, Scotland, UK \\ Tel: +441413302876 \\ Fax: +441413305602 \\ s.campo@vet.gla.ac.uk
}




\begin{abstract}
Bovine Papillomaviruses (BPVs) are oncogenic viruses. In cattle, BPV-1/2 is associated with urinary bladder cancer and BPV-4 with upper GI tract cancer. BPV E5 is a small hydrophobic protein localised in the endoplasmic reticulum (ER) and Golgi apparatus (GA). E5 is the major transforming protein of BPVs, capable of inducing cell transformation in cultured mouse fibroblasts and, in cooperation with E7, in primary bovine cells. E5-induced cell transformation is accompanied by activation of several cellular protein kinases, including growth factor receptors, and alkalinisation of endosomes and GA. We have reported that BPV E5 causes swelling and fragmentation of the GA and extensive vacuolisation of the cytoplasm. We now show that E5 from both BPV-1 and BPV-4 disturbs the actin cytoskeleton and focal adhesions in transformed bovine cells, where these morphological and behavioural characteristics are accompanied by hyperphosphorylation of the cellular phosphotyrosine kinase c-src. Both BPV-1 and BPV-4 E5 increase the motility of transformed mouse cells, but only BPV-1 E5 causes transformed mouse cells to penetrate a matrigel matrix. BPV-1 transformed mouse cells, but not BPV-4 transformed mouse cells, have hyperhpsphorylated c-src.
\end{abstract}

Key words: BPV, E5, cell transformation, cytoskeleton, focal adhesions, motility, invasion, c-src. 


\section{Introduction}

Papillomaviruses (PVs) are oncogenic viruses, which induce benign hyperproliferative lesions of the cutaneous and mucous epithelia. PV infections are usually eradicated by a cell-mediated immune response directed against viral antigens. Occasionally however in a small but sizeable minority of cases the viral lesions do not regress and progress to cancer. High risk human papillomaviruses type 16 and 18 (HPV-16 and -18) are the main causative factor in the development of cancer of the cervix uteri (zur Hausen, 2002). Bovine papillomavirus type 1 and 2 (BPV-1, BPV-2) and BPV-4 are respectively involved in carcinogenesis of the urinary bladder and of the alimentary tract in cattle (Campo, 1997). BPV-1 also induces penile papillomas that can progress to cancer (Jarrett, 1985).

The BPV-1 genome encodes three transforming proteins, E5, E6 and E7, whereas BPV-4 does not possess an E6 gene (Jackson et al., 1991). For both viruses E5 plays a major role in cell transformation (Jackson et al., 1991, Venuti \& Campo, 2002). E5 is a small hydrophobic peptide of 44 amino acid residues in BPV-1 and 42 residues in BPV-4, localised in the endomembrane compartments of the endoplasmic reticulum (ER) and Golgi apparatus (GA) of the host cell. In warts and papillomas E5 is expressed in the cytoplasm of the basal and suprabasal layers and of the spinous and squamous layers. Expression is however discontinuous (Burnett et al., 1992; Anderson et al., 1997; Araibi et al., 2004).

Despite its small size, BPV E5 has wide pleiotropic effects. It induces growth in low serum and in suspension, prevents contact inhibition, down-regulates gap junction communication (GJIC) (for reviews, see DiMaio \& Mattoon, 2001, and Venuti \& Campo, 2002, and references therein), induces swelling and fragmentation of the GA and vacuolisation of the cytoplasm (Ashrafi et al., 2002) and inhibits the expression of MHC class I on the cell surface (Ashrafi et al., 2002, Marchetti et al., 2002). BPV-1 E5 interacts with, and activates, the PDGF receptor and several other kinases, including c-src; both BPV-1 and BPV-4 E5 bind 16k ductin/subunit c, a component of the gap junction and of the V0 sector of the vacuolar $\mathrm{H}^{+}$-ATPase, and prevent the acidification of the GA. Both the down-regulation of GJIC and the alkalinisation of the endomembrane compartments are ascribed to the interaction with $16 \mathrm{k}$ ductin/subunit c and a malfunction of the V-ATPase, but the latter is not always observed (Venuti \& Campo, 2002). 
In addition to the previously described functions, here we now show that BPV-1 and BPV-4 E5 disturb the actin cytoskeleton and focal adhesions in transformed bovine cells, and increase the motility of transformed mouse cells. However, only BPV-1 E5 confers invasiveness to transformed mouse cells.

\section{Materials and Methods}

2.1 PalF cell lines: PalF are parental primary bovine foetal palate cells, PalF-control cells are partially transformed by BPV-4 E7, HPV-16 E6 and activated ras; PalF-1E5 and PalF-4E5 express either the E5 protein of BPV-1 (1E5) or BPV-4 (4E5) respectively, in addition to the other oncogenes. They have been described before (Ashrafi et al., 2000, Ashrafi et al., 2002). Please note that 4E5 was previously called 4E8. When the two BPV E5 proteins present the same characteristics they will be referred to as E5, otherwise their provenance will be specified.

2.2 NIH 3T3 lines: 3T3-pZip cells are NIH 3T3 cells harbouring empty pZipneo plasmid vector. 3T3-1E5 and 3T3-4E5 cells are NIH 3T3 cells transformed by BPV-1 E5 and BPV-4 E5 respectively and have been described before (Ashrafi et al., 2002, O'Brien V. \& Campo, 1998). All cell lines were grown in Dulbecco modified Eagles medium (DMEM) (Life Technologies, UK), $10 \%$ fetal calf serum (FCS) at $37^{\circ} \mathrm{C}$ in $5 \% \mathrm{CO}_{2}$.

2.3 Cytoimmunofluorescence. Cells were grown on glass coverslips $\left(10^{4}\right.$ cells/coverslip), washed twice with PBS, fixed by 10-min incubation at room temperature with 3\% paraformaldehyde and washed three times with PBS. They were permeabilised by 20-min incubation at room temperature with $0.5 \%$ Triton X-100 (Sigma, UK) in PBS-1\% FCS and washed three times with PBS-1\% FCS. Cells were stained with FITC- or TRITC-phalloidin (Sigma) to visualise actin fibres, or reacted with monoclonal antibody V9131 (1/400; Sigma) or monoclonal antibody PXC-10 (1/400; Sigma) to visualise vinculin or paxillin, respectively, and FITC-conjugated anti-mouse secondary antibody (1/200; Sigma). After washes with 1\% FCS in PBS, coverslips were mounted in AF1. Fluorescence was analysed using a Leica DMLB microscope or a Leica TCS SP2 confocal microscope.

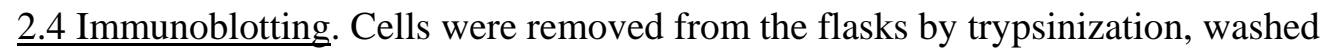
with PBS, and then lysed in lysis buffer (100 mM Tris HCl, pH 7.5, 2\% SDS, 20\% glycerol) containing protease inhibitors (Roche Diagnostic Ltd.). Insoluble material was removed by centrifugation at 20,000 g. Ten $\mu$ g of lysate were electrophoresed in 
4-12\% NuPAGE gels (Invitrogen), and proteins transferred to nitrocellulose membrane (Invitrogen) using a semidry blotting apparatus at 20V/150A for 1 hour. The membranes were blocked for $1 \mathrm{~h}$ in 5\% milk/TBS/Tween $20(0.05 \%)$ at room temperature before a $2 \mathrm{~h}$ incubation with anti-actin AB-1 (1/10,000; Merck Bioscience, UK), anti-vinculin V9131 (1/2000) and anti-paxillin PXC-10 (1/2000) antibodies (from Sigma). An antibody to GAPDH (1/2000; Abcam Ltd, UK) was used to control for the amount of protein. For detection of c-src protein, membranes were probed with anti-total c-src polyclonal antibody SC-18 (1:200; Santa Cruz Biotechnology Inc.), or anti-phopshporylated (active) c-src polyclonal antibody c$\operatorname{src}(p 416)$ (1:1000; kind gift of M. Frame). After repeated washing with TBS/Tween $20(0.05 \%)$ the membranes were incubated with either anti-mouse (for actin, vinculin, paxillin and GAPDH) or anti-rabbit (for c-src) Ig-HRP (1/2000; Amersham Pharmacia Biotech, UK) in 5\% milk/TBS/Tween 20 (0.05\%) for $1 \mathrm{~h}$ at room temperature. The membranes were washed three times with TBS/Tween 20 (0.05\%) and bound antibody was detected by enhanced chemiluminescence staining (ECL) (Amersham Pharmacia Biotech).

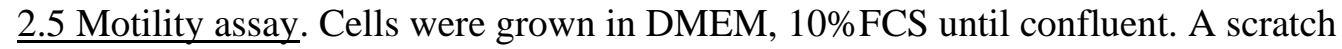
was made in the cell monolayer with a microlance 3 needle and the cell were photographed in a phase-contrast microscope at 0, 2, 4, 6 and 8 hours after scratching. The scratch width in the cell monolayer at 0 time was arbitrarily assigned a value of 1 and the width of each scratch at various time points was measured and expressed as a fraction of 1 . At least five measurements per cell line were taken.

2.6 Invasion assays. The ability of cells to invade was measured indirectly by anchorage independence assays and by matrigel invasion. The former assays were carried out as described by Pennie et al., 1993. Briefly, cells $\left(10^{5}\right)$ were added to 15 $\mathrm{ml}$ of 1\% Methocel in DMEM, 30\% FCS, plated in duplicate in bacterial Petri dishes and left at $37^{\circ} \mathrm{C}$ for 12 days before being scored and photographed. At least three assays were performed for cell line.

Inverse invasion assays were carried out as previously described (Hennigan et al., 1994) with exceptions that reduced growth factor matrigel (BD Biosciences) was used. Single cells were seeded at $2 \times 10^{4}$ per Transwell and DMEM above the matrigel contained 10\% FCS. Cells were stained with calcein AM (Scott et al., 2004). Confocal microscopy and quantitation was largely as previously described (Hennigan et al., 1994), with exceptions that a positive pixel is defined as one with an intensity 
value greater than operator-defined background and that optical sections were scanned at $3 \mu \mathrm{m}$ intervals. Only cells in the $9 \mu \mathrm{m}$ section or above were considered invasive for quantitation purposes. A compound image of these sections was quantified (Hennigan et al., 1994) then normalised to the value obtained from the corresponding $0 \mu \mathrm{m}$ section as a "loading" control. Overall results were determined from three separate assays performed with duplicate samples in each, with the exception of 3T34E5 cells, which were only included in two assays. Up to 6 separate serial sections were quantified for each sample.

\section{Results}

3.1 E5 disturbs and collapses the actin cytoskeleton in PalF cells. PalF-1E5 and PalF4E5 cells have a very disturbed morphology: they are highly vacuolated and have a swollen, misshapen and often fragmented GA (see Figure 4 of Faccini et al., 1996, Figure 1 of Ashrafi et al., 2000 and Figure 1 of Ashrafi et al, 2002).

To see if E5 expression affected other cell structures, we stained the PalF cell lines for actin fibres with FITC- or TRITC-phalloidin which stains the cytoskeleton. Analysis of the cells revealed that cells expressing E5 had long pseudopods, lamellipods and pronounced ruffles and a profoundly disturbed actin cytoskeleton, with shortened actin fibres that did not appear to reach the cell membrane (Figure 1A). The shortening of the actin fibres was not observed in PalF cells expressing 4N17S and 4E5T, non-transforming mutants of BPV-4 E5, whereas it was present in cells expressing 4N17A, a hyper-transforming mutant of the protein (Figure 1A). These differences in cell morphology were not due to differences in expression of the various E5 proteins as both wild type E5 and its mutants were expressed at comparable levels (O’Brien V et al., 1999). This confirmed that the abnormal morphology of the actin cytoskeleton in E5-expressing cells was due to the transforming action of E5. Furthermore, no obvious abnormalities were observed in the parental non transformed cells or in the transformed PalF-control cells (Figure 1A; Table 1).

3.2 E5 disturbs focal adhesions in PalF cells. The fibres of the actin cytoskeleton connect with focal adhesions (FA), structures that allow interaction between cells and substrate (Geiger et al., 2001). Vinculin and paxillin are both component of FA 
(Geiger et al., 2001). To determine whether FA were also affected by E5, cells were stained with antibodies against vinculin or paxillin. In the parental PalF and PalFcontrol cells, vinculin was clearly located in FA at the periphery of the cell (Figure 1B). In the PalF-1E5 and PalF-4E5 cells vinculin was detected in internal structures, while small chain-like structures similar to focal complexes (Geiger et al., 2001) were sometimes observed at the cell periphery (Figure 1B and inset; Table 1). Growing PalF-E5 cells on fibronectin, which, being a ligand for integrins, induces formation of FA, did not lead to the appearance of FA on the cell surface (data not shown). FA therefore appeared disturbed in PalF-E5 cells. When PalF cells were co-stained for actin and vinculin, vinculin was found at the end of the shortened actin fibres in E5 cells (Figure 1C), indicating that the interaction between vinculin and actin fibres was maintained but not on the cell surface. In PalF cells expressing non transforming mutants of BPV-4 E5, the FA resembled those of parental and control cells, whereas in PalF cells expressing the hyper-transforming mutant they resembled those of PalFE5 cells (Figure 1C). Similar results were obtained with paxillin, another component of FA (data not shown).

\subsection{E5 does not induce degradation of actin or FA components. To see if the} disruption of the actin fibres and the disassembly of the FA were due to E5-induced degradation of actin, vinculin or paxillin, as observed for the heavy chain of the major histocompatibility complex (MHC) class I (Ashrafi et al., 2002; Marchetti et al., 2002), cell lysates from the PalF cell lines were analysed for these proteins. No noticeable differences were seen between control cells and E5-expressing cells (Figure 2). Moreover the pattern of actin fibres and of FA did not change when E5expressing cells were incubated with the proteasome inhibitor MG-132 or the calpain inhibitors I and II (calpain degrades FA proteins) (data not shown), confirming that the observed interference with these structures is not due to protein degradation.

3.4 E5 enables PalF cells to grow independently of substrate. Adhesion to substrate is controlled by FA and disruption of FA promotes cell growth independent of substrate (Carragher and Frame, 2004). We have already reported that both PalF and NIH 3T3 cells expressing BPV E5 are capable of anchorage-independent growth (O'Brien V et al., 1999, O'Brien V. \& Campo, 1998). We confirm that this is indeed the case: PalF1E5, PalF-4E5 and PalF-4N17A cells grew in methocel, whereas neither parental 
PalF, control or PalF-4E5T cells were capable of doing so (Figure 3; Table 1). The disruption of FA brought about by E5 is likely to contribute to the ability of the cells to grow in an anchorage independent fashion.

3.5 NIH 3T3 cells expressing BPV E5 are more motile. The cytoskeleton and the FA regulate cell shape and cell motility (Geiger et al., 2001). Given the profusion of pseudopods, lamellipods and membrane ruffles and the absence of FA in PalF-1E5 and PalF-4E5 cells, we sought to analyse their motility in the scratch wound assay. However, the assay could not be performed with the PalF cell lines because the cell monolayers would lift and float instead of scratching (Table 1). Therefore we decided to analyse NIH 3T3 cells transformed by BPV E5 (O’Brien V \& Campo, 1998;

O'Brien V et al., 2001). First we analysed the status of the actin cytoskeleton of these cells, as we did for the PalF cell lines. Following staining with FITC-phalloidin, the actin cytoskeleton was normally organised in 3T3-pZip cells, but was collapsed in the 3T3-E5 cells, with actin located cortically and long pseudopodes (Figure 1A; Table 1). The morphology of the 3T3-E5 cells was however less disturbed that that of PalFE5 cells. Despite the collapse of the actin cytoskeleton, as in the PalF cells, there were no differences in the levels of actin between control and E5 cells (data not shown), and no disruption of FA was observed in the 3T3-E5 cells with either the paxillin or the vinculin antibody (data not shown; Table 1). In the scratch assay, the 3T3-E5 cells repopulated and closed the wound faster than control cells (Figure 4A,B; Table 1), indicating that BPV E5 can increase cell motility, at least in NIH 3T3 cells. Increase motility however appears to be independent of the status of FA in NIH 3T3 cells.

3.6 NIH 3T3-1E5 cells are more invasive. Next we wished to analyse whether, in addition to increased motility, E5 transformed cells would also be more invasive. To this end we employed the three-dimensional matrigel invasion assay (Hennigan et al., 1994, Scott et al., 2004). Both control and PalF-E5 cells died in the matrigel and therefore their invasion ability could not be assessed (Table 1). Thus, as for the motility assay, we performed the test on the NIH 3T3 cells. Parental 3T3-pZip cells did not invade the matrigel, whereas 3T3-1E5 cells were capable of invasion and were detected at least $9 \mu \mathrm{m}$ from the filter (Figure 5). Surprisingly however, 3T3-4E5 cells were not capable of invading and were not detected beyond the upper surface of the 
filter (Figure 5; Table 1). The inability of 3T3-4E5 cells to invade the matrigel was not due to poor E5 expression, as E5 was expressed at detectable levels (O'Brien V et al., 2001), or to death as the cells were detected on the filter (Figure 5).

3.7 c-src is hyperphosphorylated in E5-expressing cells. The cellular protein c-src is a tyrosine kinase that modulates the actin cytoskeleton and cell adhesions (Frame et al., 2002). Activation of the catalytic activity of c-src through phosphorylation initiates a signal transduction cascade that induces adhesion turnover, actin re-modelling, cell migration and invasion (Frame, 2002, Frame, 2004, Frame \& Brunton, 2002). Given the ability of PalF E5 cells to grow in suspension and the disrupted state of their cytoskeleton and FA, and the greater motility of 3T3-1E5 and 3T3-4E5 cells and invasion properties of the 3T3-1E5 cells, we examined the phosphorylation status of c-src in these cells. We found that c-src was more highly phosphorylated in transformed PalF-1E5 and PalF-4E5 cells and in 3T3-1E5 cells than in the parental or control cells (Figure 6A, B; Table 1). On the contrary, c-src was hypophosphorylated in 3T3-4E5 cells which were incapable of invasion. Thus, a precise correlation between c-src activation and cell morphology and behaviour could not be established.

Cytoskeletal rearrangements and cell motility can be controlled by the small GTPase family members (Nobes \& Hall, 1999). We analysed levels of RhoA, Rac and Cdc42 in both PalF and 3T3 E5-expressing cells but did not find any difference with control cells (data not shown). We were unable to detect activated Rho GTPases in our cellular samples by using specific pull-down assays, and therefore the activation status of the small GTPases remains to be elucidated.

Table 1 shows a summary of the characteristics of PalF and 3T3 cells.

\section{Discussion}

We describe here further characteristics of morphological cell transformation brought about by the E5 oncoproteins from one cutaneous (BPV-1) and one mucosal BPV (BPV-4) in two different cell systems, primary bovine PalF cells (the natural host of BPV) and established murine NIH 3T3 cells. While PalF cells need several viral (E5, E6 and E7) and cellular (activated ras) oncoproteins to achieve transformation (Pennie 
et al., 1993, O’Brien V et al., 1999), mouse NIH 3T3 cells can be easily transformed by a variety of agents, including BPV E5 (Schiller et al., 1986; O’Brien V \& Campo, 1998). Despite these differences, the use of the two different cell systems has provided information that could not be obtained in one system alone.

\subsection{E5 and PalF cell morphology. Expression of either BPV-4 or BPV-1 E5 brings} about profound modifications in the actin cytoskeleton, with "shortened" actin fibres, without any noticeable reduction in the overall amount of actin. These cytoskeletal rearrangements are accompanied by increased formation of lamellipodia, membrane ruffles and filopodia.

Both E5 proteins induce also the relocation, but not degradation, of vinculin and paxillin (both components of FA) at the end of the shortened actin fibres. This destabilisation of FA, in addition to E5-induced up-regulation of cyclin A (O'Brien V et al., 1999), is likely to underlie the ability of E5-transformed cells to grow independently of anchorage to the substrate (O’Brien V et al., 1999; Ashrafi et al., 2000).

The cytoskeleton and FA are not the only cell structures affected by E5 in PalF cells. The Golgi apparatus (GA) is enlarged and often fragmented, and the cytoplasm is highly vacuolated (Faccini et al., 1996; Ashrafi et al., 2000; Ashrafi et al., 2002). We have attributed these modifications of the endomembrane compartments to their alkalinisation brought about by a malfunction of the $\mathrm{H}^{+} \mathrm{V}$-ATPase due to the interaction between E5 and 16k subunit c, as similar changes in the GA and cytoplasm are caused in control cells by the ionophore monensin, which inhibits the proton pump (Marchetti et al., 2002). However, treatment of PalF control cells with monensin did not cause changes in FA, and the changes in actin fibres were dissimilar to those observed in E5 cells (data not shown). We therefore conclude that the rearrangement of the actin cytoskeleton and the destabilisation of the FA are caused by E5 through a different pathway(s) than the targeting of the proton pump.

PalF cells transformed by the complete BPV-4 genome and activated ras also have a distorted shape with enlarged cytoplasm and vacuoles (Jaggar et al., 1990). Although 
not experimentally proven, it is reasonable to assume that these changes are due, at least in part, to the E5 protein.

$\underline{4.2}$ E5, cell motility and invasiveness in NIH 3T3 cells. BPV E5 induces anchorage independence in PalF cells as described earlier (Pennie et al., 1993; O’Brien V et al., 1999) and confirmed here. Despite their ability to grow when deprived of substrate, the PalF-E5 cells were not capable of surviving in the matrigel and thus their invasion ability could not be assessed. The reason for the failure of the PalF E5 cells to survive in the matrigel may reside in their inability to stay alive in the absence of contact with surrounding cells. Likewise, we could not establish the effects of E5 on motility in PalF cells because of the lifting of the monolayer when scratched. Given the impossibility to assess the motility and invasiveness of PalF cells, we decided to investigate these properties in NIH 3T3 cells transformed by either BPV-1 or BPV-4 E5 (O’Brien V and Campo, 1998, O'Brien V et al., 2001). The analysis of these transformed cells proved fruitful and in addition revealed differences between BPV-1 E5 and BPV-4 E5. While both 3T3-1E5 cells and 3T3-4E5 cells had a disturbed actin cytoskeleton and showed enhanced motility, only 3T3-1E5 cells proved invasive, suggesting a greater transformation potential of BPV-1 E5 than BPV-4 E5 in NIH 3T3 cells.

4.3 Activation of c-src. The cellular tyrosine kinase c-src modulates the actin cytoskeleton and cell adhesions (Frame et al, 2002). Phosphorylation of c-src activates a signal transduction cascade that induces adhesion turnover, actin remodelling, cell migration and invasion (Frame, 2002, Frame \& Brunton, 2002). BPV1 E5 activates c-src in NIH 3T3 cells (Suprynowicz et al., 2002) and, in agreement with these observations, we found that c-src is hyperphosphorylated (activated) in PalF-1E5 and PalF-4E5 cells and in 3T3-1E5 cells. The activation of c-src may be due to the E5-induced alkalinisation of the GA (Schapiro et al., 2000), as speculated by Suprynowicz et al. (2002) and may contribute to the transformed status of the cells observed here. However, c-src is not hyperphosphorylated in 3T3-4E5 cells, highlighting another difference between BPV-1 E5 and BPV-4 E5. As there is not a clear-cut correlation between c-src activation and cell morphology and behaviour, the precise consequence of c-src activation cannot be unequivocally established. The reason why BPV-4 E5 induces phosphorylation of c-src in PalF cells but not in NIH 
3T3 cells is not known. It is to be noted however that BPV-4 E5 is not alone in exhibiting cell-specific effects: the oncoprotein v-fos easily transforms rodent fibroblasts but not human cells, although it makes the latter invasive (Scott et al., 2004, and references therein).

4.4 Cell morphology and viral proteins. It is interesting that the Nef protein of HIV, with which E5 shares the ability to bind components of the $\mathrm{H}^{+} \mathrm{V}$-ATPase and to down-regulate surface MHC class I (Kasper \& Collins, 2003, Lu et al., 1998), also induces rearrangements of the actin cytoskeleton and the formation of lamellipodia and filopodia (Fackler et al., 1999). Expression of SV40 small t in epithelial cells brings about similar morphological and structural changes (Nunbhakdi-Craig et al., 2003), including the formation of numerous giant cells, as in our PalF system (Ashrafi et al., 2000, Faccini et al., 1996). The changes in cell shape and cytoskeleton observed in these three different viral protein systems resemble those associated with activation of small GTPases, particularly Rac (Nobes \& Hall, 1999). Indeed this is the case for Nef (Fackler et al., 1999), and both Rac and Cdc42 are over-expressed in small texpressing cells (Nunbhakdi-Craig et al., 2003). Like Nunbhakdi-Craig et al. (2003), we could not detect activation of the small GTPases, but, unlike those authors, did not find any change in their level of expression (data not shown) and are therefore unable to conclude that E5 activates them.

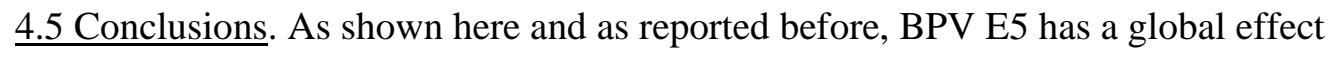
on the morphology and behaviour of PalF cells: cells are vacuolated with a distended and fractured Golgi apparatus, have a rearranged actin cytoskeleton and disassembled focal adhesions, grow in low serum and independently of substrate, and protein traffic to the cell periphery is disturbed. Furthermore E5 affects several signal transduction pathways and cell cycle regulators. The small size of E5 suggests that these multiple effects are due to a critical interaction(s) between E5 and a crucial cellular partner(s). 


\section{Acknowledgements}

We are grateful to Prof Frame and her colleagues for the antibody c-src(p416) and for critical discussions. We thank Dr Morgan for critically reading the manuscript. The work was funded by Cancer Research UK and the Medical Research Council. MSC is a Fellow of CR UK. 


\section{References}

Anderson R. A., Scobie L., O’Neil B. W., Grindlay G. J. \& Campo M. S. (1997). Viral proteins of bovine papillomavirus type 4 during the development of alimentary canal tumours. Veterinary $\mathrm{Jl} \mathbf{1 5 4}, 69-78$.

Araibi, E.H., Marchetti, B., Ashrafi, G. H. \& Campo, M. S. (2004) Down-regulation of MHC class I in bovine papillomas. J Gen Virol 85, 2809-2814.

Ashrafi, G. H., Pitts, J. D., Faccini, A., McLean, P., O'Brien, V., Finbow, M. E. \& Campo, S. (2000). Binding of bovine papillomavirus type 4 E5 to ductin (16K proteolipid), down-regulation of gap junction intercellular communication and full cell transformation are independent events. J Gen Virol, 81, 689-694.

Ashrafi, G. H., Tsirimonaki, E., Marchetti, B., O'Brien, P. M., Sibbet, G. J., Andrew, L. \& Campo, M. S. (2002). Down-regulation of MHC class I by bovine papillomavirus E5 oncoproteins. Oncogene 21, 248-259.

Burnett, S., Jaresborg, N. \& DiMaio D. (1992) Localization of bovine papillomavirus type $1 \mathrm{E} 5$ protein to transformed basal keratinocytes and permissive differentiated cells in fibropapilloma tissue. Proc Natl Acad Sci.89, 56655669.

Campo, M. S. (1997). Bovine papillomavirus and cancer. Veterin. J. 154, 175-188.

Carragher N.O. \& Frame M.C. (2004) Focal adhesion and actin dynamics: a place where kinases and proteases meet to promote invasion. Trends Cell Biol. 14, 241-249.

DiMaio, D. \& Mattoon, D. (2001). Mechanisms of cell transformation by papillomavirus E5 proteins. Oncogene 20, 7866-7873.

Faccini, A. M., Cairney, M., Ashrafi, G. H., Finbow, M. E., Campo, M. S. \& Pitts, J. D. (1996). The bovine papillomavirus type 4 E8 protein binds to ductin and causes loss of gap junctional intercellular communication in primary fibroblasts. $J$ Virol 70, 9041-9045.

Fackler, O., Luo, W, Geyer, M, Alberts, AA, Peterlin, BM. (1999). Activation of Vav by Nef induces cytoskeletal rearrangements and dowstream effector functions. Molecular Cell 3, 729-739.

Frame, M. C. (2002). Src in cancer: deregulation and consequences for cell behaviour. Biochimica et Biophysica Acta (BBA) - Reviews on Cancer 1602, 114-130.

Frame, M. C. (2004). Newest findings on the oldest oncogene; how activated src does it. $J$ Cell Sci 117, 989-998.

Frame, M. C. \& Brunton, V. G. (2002). Advances in Rho-dependent actin regulation and oncogenic transformation. Current Opinion in Genetics \& Development 12, 36-43.

Frame, M. C., Fincham, V. J., Carragher, N. O. \& Wyke, J. A. (2002). v-Src's hold over actin and cell adhesions. Nat Rev Mol Cell Biol. 3, 233-245.

Geiger, B., Bershadsky, A., Pankov, R. \& Yamada, K. M. (2001). Transmembrane extracellular matrix--cytoskeleton crosstalk. Nat Rev Mol Cell Biol. 2, 793805.

Hennigan, R. F., Hawker, K. L. \& Ozanne, B. W. (1994). Fos-transformation activates genes associated with invasion. Oncogene 9, 3591-600.

Jackson, M. E., Pennie, W. D., McCaffery, R. E., Smith, K. T., Grindlay, G. J. \& Campo, M. S. (1991). The B subgroup bovine papillomaviruses lack an identifiable E6 open reading frame. Mol Carcinog 4, 382-387.

Jaggar R. T., Pennie W. D., Smith K. T., Jackson M. E. \& Campo M. S. (1990) Cooperation between bovine papillomavirus type 4 and ras in the 
morphological transformation of primary bovine fibroblasts. J. Gen. Virol., 71, 3041-3046.

Jarrett, W. F. H. (1985). The natural history of bovine papillomavirus infections. Advances in Viral Oncology 5, 83-102.

Kasper, M. R. \& Collins, K. L. (2003). Nef-Mediated Disruption of HLA-A2 Transport to the Cell Surface in T Cells. J. Virol. 77, 3041-3049.

Lu, X., Yu, H., Liu, S. H., Brodsky, F. M. \& Peterlin, B. M. (1998). Interactions between HIV1 Nef and vacuolar ATPase facilitate the internalization of CD4. Immunity 8, 647-656.

Marchetti, B., Ashrafi, G. H., Tsirimonaki, E., O'Brien, P. M. \& Campo, M. S. (2002). The papillomavirus oncoprotein E5 retains the major histocompatibility class I in the Golgi apparatus and prevents its transport to the cell surface. Oncogene 21, 7808-7816.

Nobes, C. D. \& Hall, A. (1999). Rho GTPases Control Polarity, Protrusion, and Adhesion during Cell Movement. J. Cell Biol. 144, 1235-1244.

Nunbhakdi-Craig, V., Craig, L., Machleidt, T. \& Sontag, E. (2003). Simian Virus 40 Small Tumor Antigen Induces Deregulation of the Actin Cytoskeleton and Tight Junctions in Kidney Epithelial Cells. J. Virol. 77, 2807-2818.

O'Brien V, Ashrafi, G. H., Grindlay, G. J., Anderson, R. \& Campo, M. S. (1999). A mutational analysis of the transforming functions of the E8 protein of bovine papillomavirus type 4. Virology 255, 385-394.

O'Brien, V., Grindlay, J. G. \& Campo, M. S. (2001). Cell transformation by the E5 protein of bovine papillomavirus type 4: p27kip1, elevated through increased protein synthesis, is sequestered by cyclin D1-CDK4 complexes. J. Biol. Chem. 276, 33861-33868.

O'Brien, V. \& Campo, M. S. (1998). BPV-4 E8 transforms NIH 3T3 cells, upregulates cyclin A and cyclin A-associated kinase activity and de-regulates expression of the cdk inhibitor p27KIP1. Oncogene 17, 293-301.

Pennie, W.D., Grindlay, G.J., Cairney, M. \& Campo, M.S. (1993). Analysis of the transforming functions of BPV-4. Virology, 193, 614-628.

Schapiro, F., Sparkowski, J., Adduci, A., Suprynowicz, F., Schlegel, R. \& Grinstein, S. (2000) Golgi alkalinization by the papillomavirus E5 oncoprotein. $J$ Cell Biol. 148, 305-315.

Schiller J. T., Vass W. C., Vousden K.H. \& Lowy D. R. (1986) E5 open reading frame of bovine papillomavirus type 1 encodes a transforming gene. $J$ Virol. 57, 1-6.

Scott, L. A., Vass, J. K., Parkinson, E. K., Gillespie, D. A. F., Winnie, J. N. \& Ozanne, B. W. (2004). Invasion of normal human fibroblasts induced by v-Fos is independent of proliferation, immortalization and the tumor suppressors p16 ${ }^{\mathrm{INK} 4 \mathrm{a}}$ and p53. Mol Cell Biol 24, 1540-1559.

Suprynowicz, F. A., Baege, A., Sunitha, I. \& Schlegel, R. (2002). c-Src activation by the E5 oncoprotein enables transformation independently of PDGF receptor activation. Oncogene 21, 1695-1706.

Venuti, A. \& Campo, M. S. (2002). The E5 protein of Papillomaviruses. In Progress in Medical Virology: Papillomaviruses. DJ McCance editor, pp141-162.

zur Hausen, H. (2002). Papillomaviruses and cancer: from basic studies to clinical application. Nature Reviews, Cancer 2, 342-350. 
8. Table 1. Characteristics of bovine and murine cells expressing BPV E5.

\begin{tabular}{|l|l|l|l|l|l|l|}
\hline & $\begin{array}{l}\text { PalF control } \\
\text { cells }\end{array}$ & PalF-1E5 & PalF-4E5 & $\begin{array}{l}\text { 3T3 control } \\
\text { cells }\end{array}$ & 3T3-1E5 & 3T3-4E5 \\
\hline $\begin{array}{l}\text { Disturbed } \\
\text { cytoskeleton }\end{array}$ & no & yes & yes & no & yes & yes \\
\hline $\begin{array}{l}\text { Mis-located } \\
\text { vinculin/paxillin }\end{array}$ & no & yes & yes & no & no & no \\
\hline $\begin{array}{l}\text { Anchorage } \\
\text { independence }\end{array}$ & no & yes & yes & no & yes* & yes* \\
\hline Motility & $\mathrm{nd}^{+}$ & $\mathrm{nd}^{+}$ & $\mathrm{nd}^{+}$ & no & yes & yes \\
\hline Invasion & no $^{\$}$ & $\mathrm{nd}^{\$}$ & $\mathrm{nd}^{\$}$ & no & yes & no \\
\hline pY-c-src & no & yes & yes & no & yes & no \\
\hline
\end{tabular}

*data from O’Brien V and Campo, 1998; ${ }^{+}$could not be determined because of monolayer lifting; ${ }^{\$}$ could not be determined because of cell death in matrigel. 


\section{Figure Legends}

Figure 1. Morphological changes in cells expressing BPV-1 or BPV-4 E5 proteins. A, PalF-E5 cells and PalF-4E5N17A cells have shortened actin fibres and large lamellipodia protrusions. Parental PalF cells, control cells and cells expressing nontransforming mutants of BPV-4 E5 have a well organised cytoskeleton. 3T3-E5 cells have a collapsed actin cytoskeleton, while control 3T3Zip cells have a well organised cytoskeleton. Cells were stained with FITC-phalloidin (green) or TRITC-phalloidin (red) and analysed using a Leica DMLB microscope. TRITC-phalloidin was used to permit double fluorescence analysis (see panel C). The white line in the 4E5N17A panel delineate the outer margin of the cell. The large size of the PalF-1E5, PalF-4E5 and PalF-4E5N17A cells precludes the imaging of a larger field without loss of resolution, but all PalF E5 cells present a morphology similar to the one shown here for single cells. Magnification 400x. B, PalF-E5 and PalF-4E5N17A cells do not have focal adhesions on their surface. Cells were stained with mAb V9131 (1/400) to visualise vinculin, and FITC-conjugated anti-mouse secondary antibody (1/200). Cells were analysed using a Leica DMLB microscope. The boxed areas in the 1E5 and 4E5 panels are enlarged in the panels below to show the absence of focal adhesions on the cell surface and the presence of focal complex-like structures. An equivalent area is enlarged for PalF-4E5N17A cells. Magnification 400x. C, PalF-E5 cells have vinculin at the tip of the short actin fibres. Cells were stained for vinculin as in B, and with TRITC-phalloidin to visualise actin fibres as in A. Cells were analysed in a Leica TCS SP2 confocal microscope and images merged using the accompanying software. Magnification 400x. The images of 1E5 and 4E5 cells are at higher magnification (800x) to show the interaction between vinculin and actin at the tip of the shortened actin fibres. Likewise, the images of 4E5N17S and 4E5T are at higher magnification (800x) to show the presence of vinculin at the cell periphery. The white lines in the $1 \mathrm{E} 5$ and 4E5 panels delineate the outer margin of the cells. For cell nomenclature, see Materials and Methods.

Figure 2. Actin and focal adhesion components in PalF-E5 cells. E5 does not induce the degradation of actin, vinculin or paxillin. Membranes with proteins from PalF cells were probed with anti-actin mAb AB-1 (1/20,000), anti-vinculin mAb V9131 (1/2000) and anti-paxillin mAb PXC-10 (1/2000) and then anti-mouse Ig-HRP (1/2000). Bound antibody was detected by enhanced chemiluminescence staining. 
Figure 3. PalF-E5 cells and PalF-4E5N17A cells, but not PalF-4E5T or control cells, grow in Methocel. Cells were grown in 1\% Methocel in DMEM, 30\% FCS and photographed after 12 days of growth (x40). At least three assays were performed for cell line. Numbers in the panels indicate the average \% (+/-SD) of total cells seeded into each plate on day $0\left(10^{5}\right)$.

Figure 4. 3T3-E5 cells are more motile. A, Cell monolayers were scratched (wounded) and the scratch width at 0 time was arbitrarily assigned a value of 1 and the width of each scratch at various time points was measured and expressed as a fraction of 1 . At least five measurements per cell line were taken. $\mathbf{B}$, examples of wound healing of 3T3-pZip, 3T3-1E5 and 3T3-4E5 cells. The black vertical lines indicate the maximum width of the wound. Images were taken at $0 \mathrm{hr}$ and at $6 \mathrm{hr}$. Figure 5. 3T3-1E5 cells are invasive. A, Confocal microscope images of calceinstained cells in the invasion assay. The upper row shows cells on the underside of the filter and the lower row shows the corresponding compound images of transverse sections (sections 4 and above), showing cells that have invaded greater than $9 \mu \mathrm{m}$ into the matrigel. B, Quantitation of invading cells. The units for the y-axis are percent positive pixels after normalization for loading (see Materials and Methods for details).

Figure 6. C-src is hyperphosphorylated in E5-expressing cells. A, PalF cells. Top panel, membranes with cell proteins were probed with anti-phosphorylated (active) csrc polyclonal antibody c-src(p416) (1:1000) or anti-total c-src polyclonal antibody SC-18 (1:200), and then anti-rabbit Ig-HRP (1/2000). Bound antibody was detected by enhanced chemiluminescence staining. Bottom panel, the western blots were scanned in a MagicScan and quantified using the Image Quant 5.3 Software (Molecular Dynamics). The ratio of phosphorylated c-src over total c-src is shown. The experiment was repeated two times with essentially identical results. B, NIH 3T3 cells. Top panel, membranes with cell proteins were probed as in A top panel, and the western blots were scanned and quantified as in A bottom panel. 
A
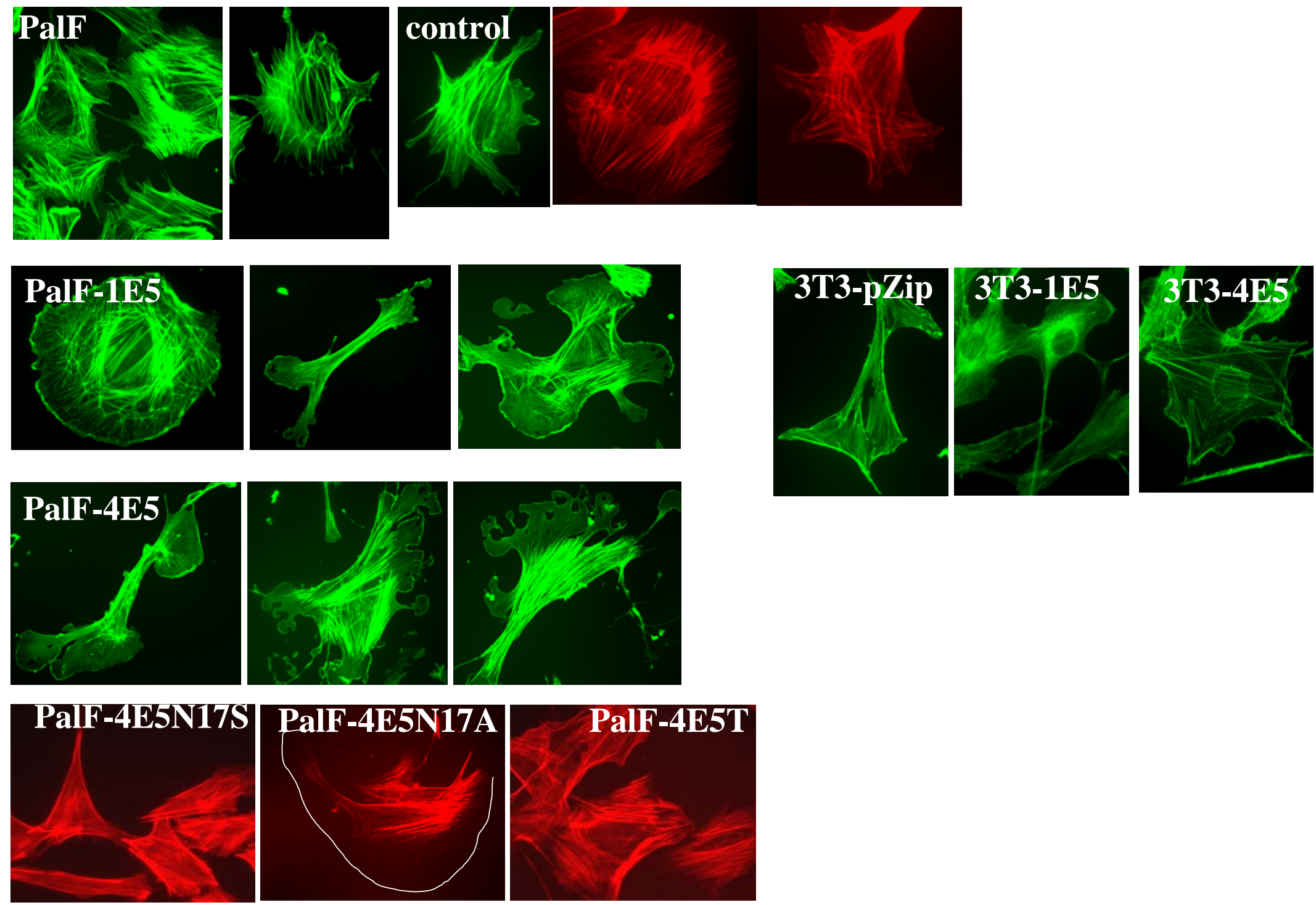

Tsirimonaki et al., Figure 1A 
B
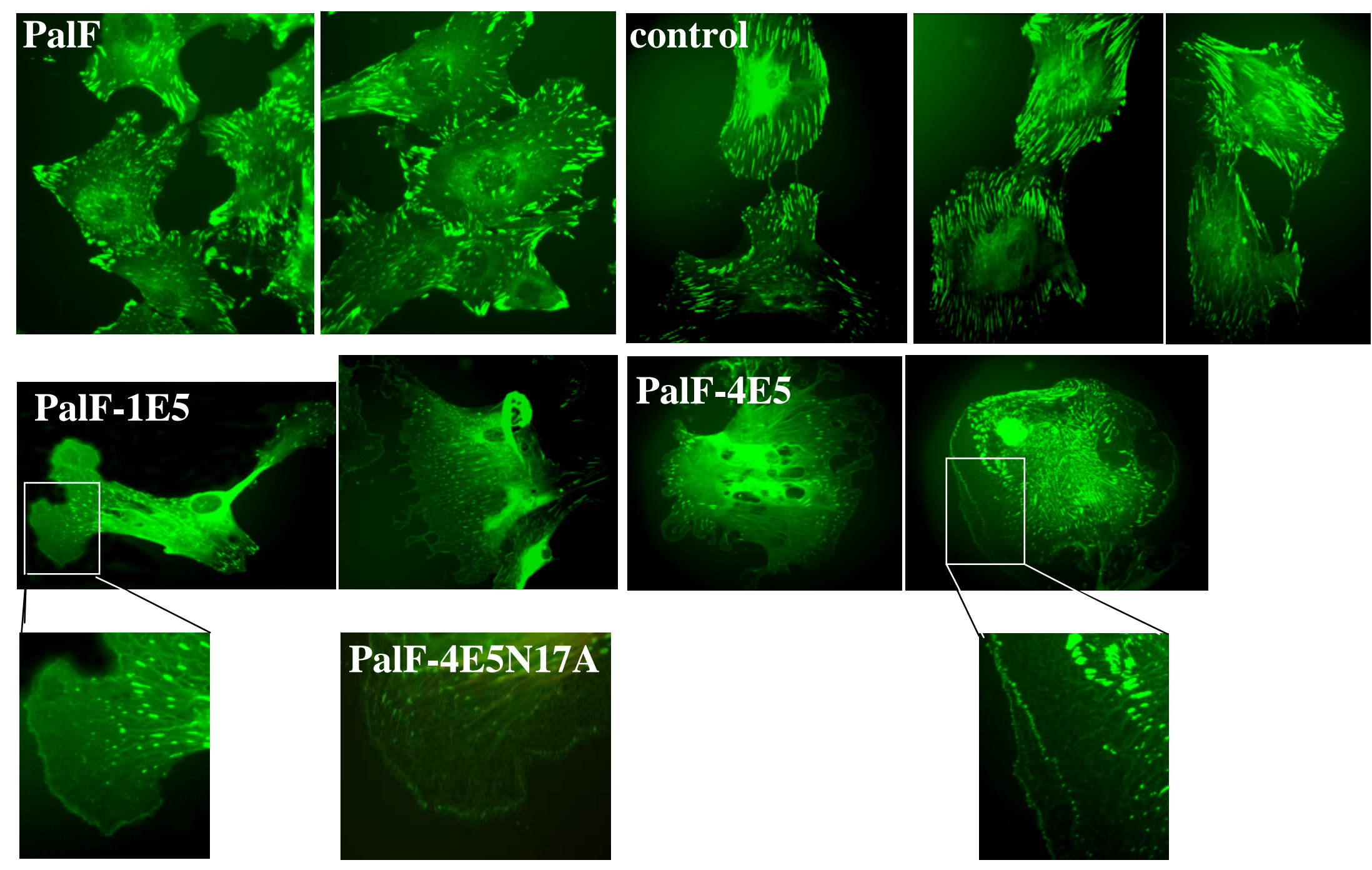

Tsirimonaki et al., Figure 1B 

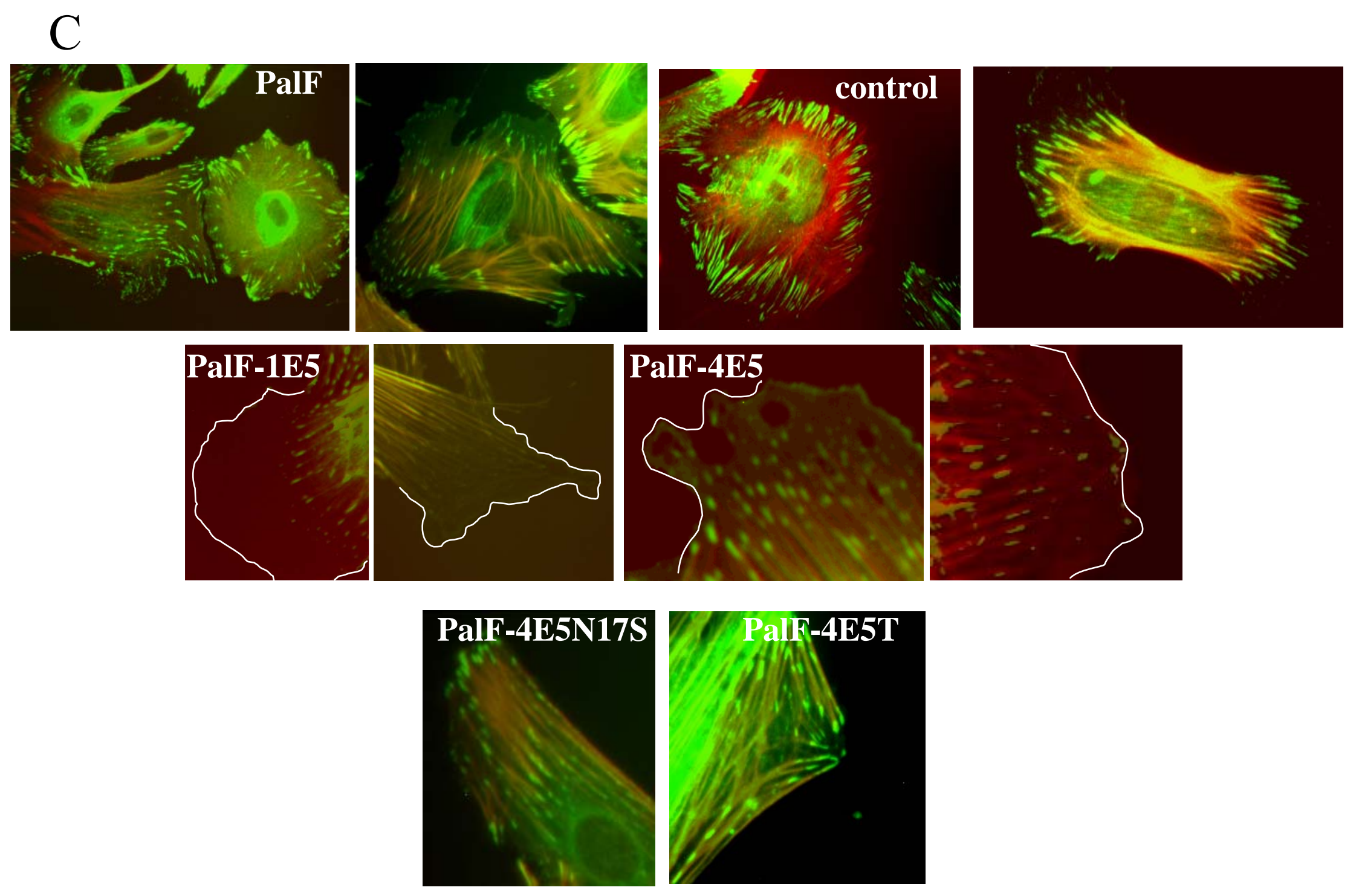

Tsirimonaki et al., Figure 1C 


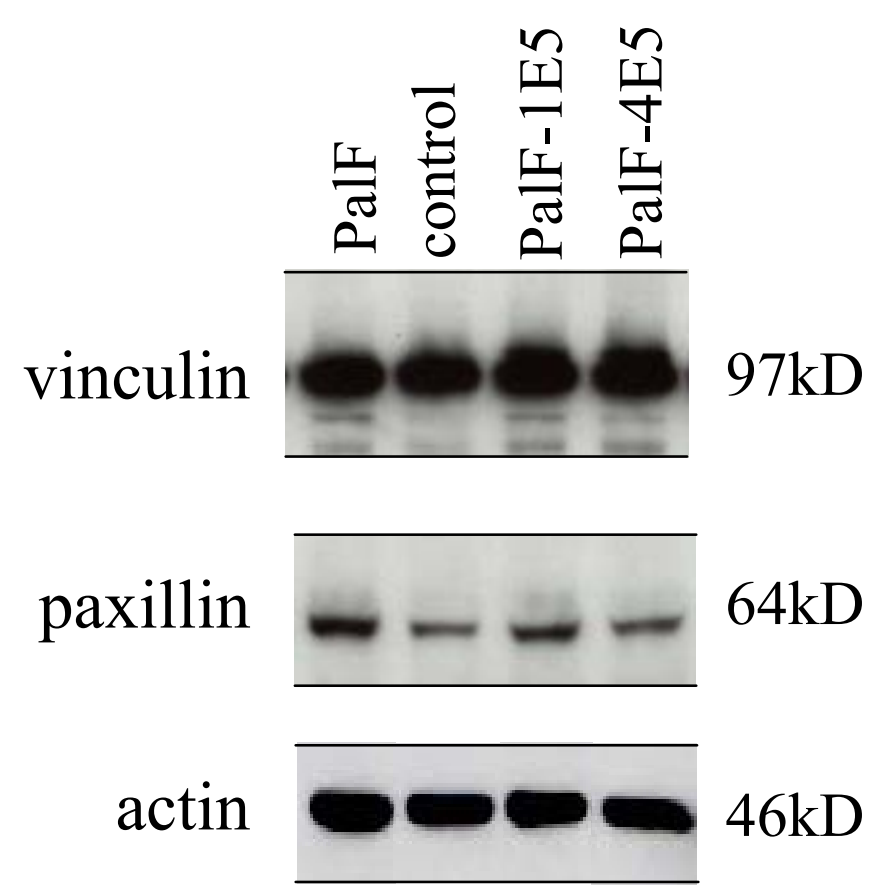

Tsirimonaki et al., Figure 2 


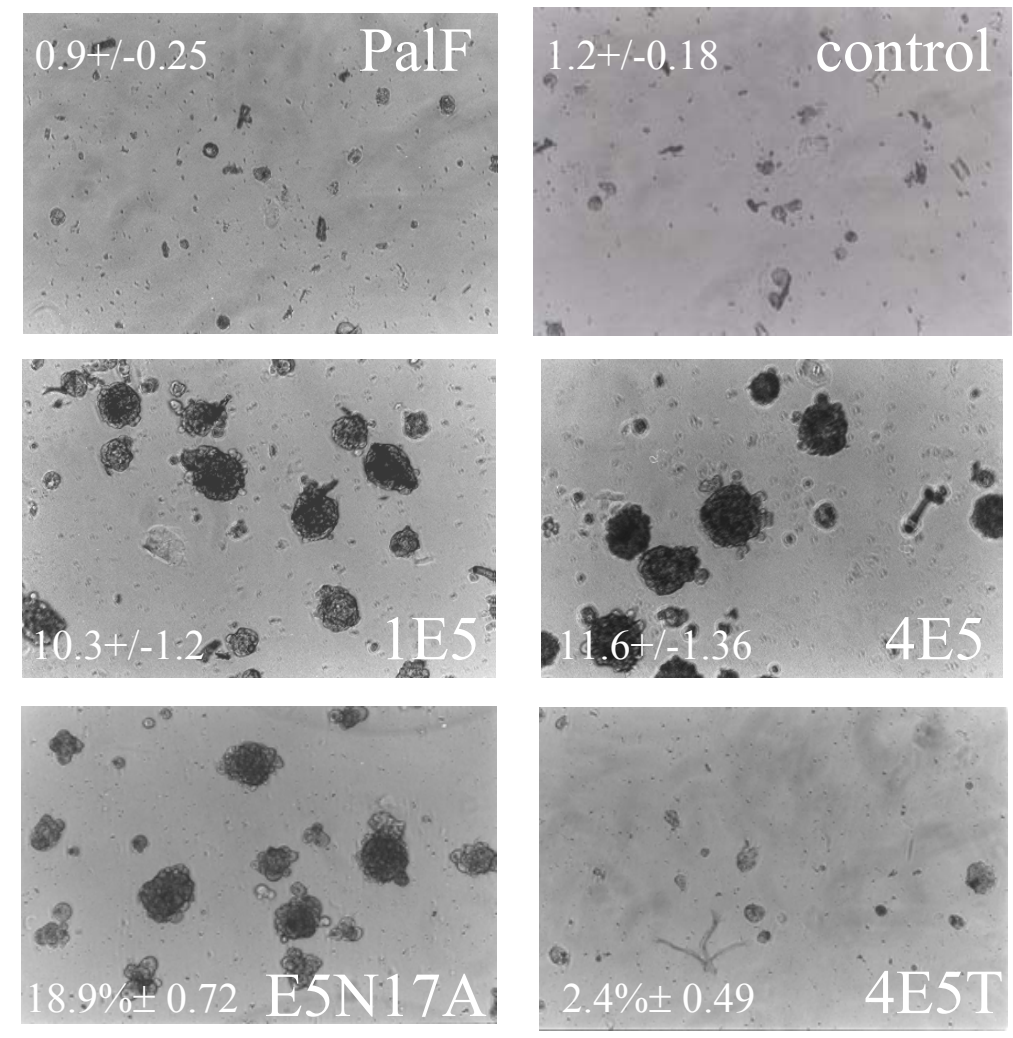

Tsirimonaki et al., Figure 3 
Tsirimonaki et al., Figure 4

A

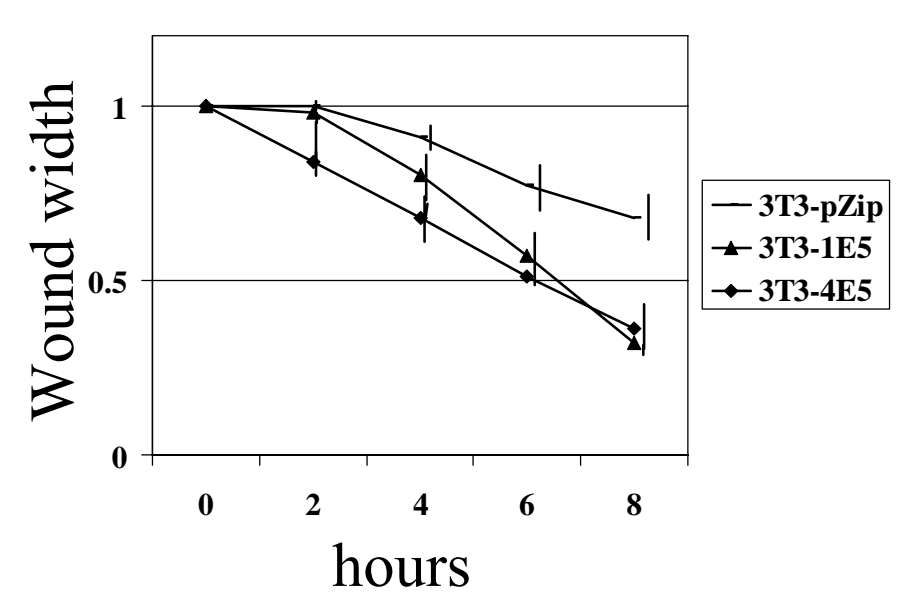

B

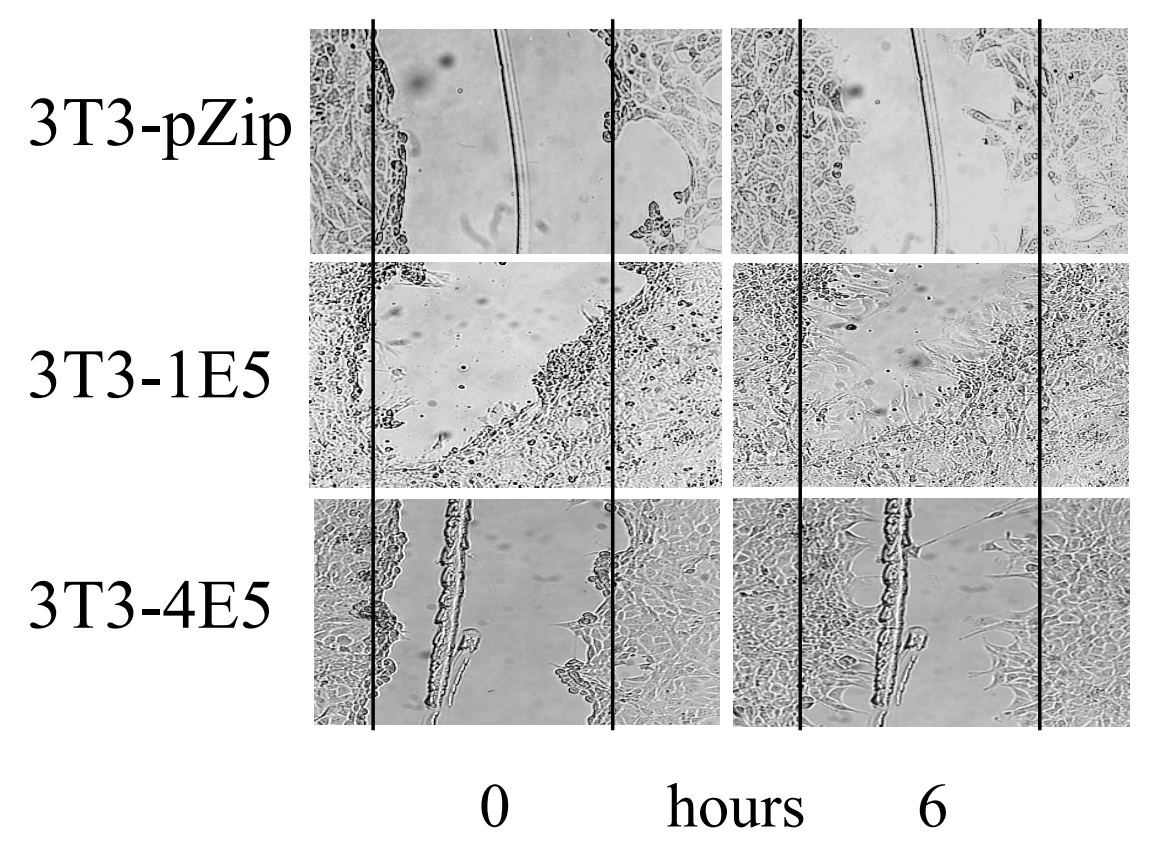




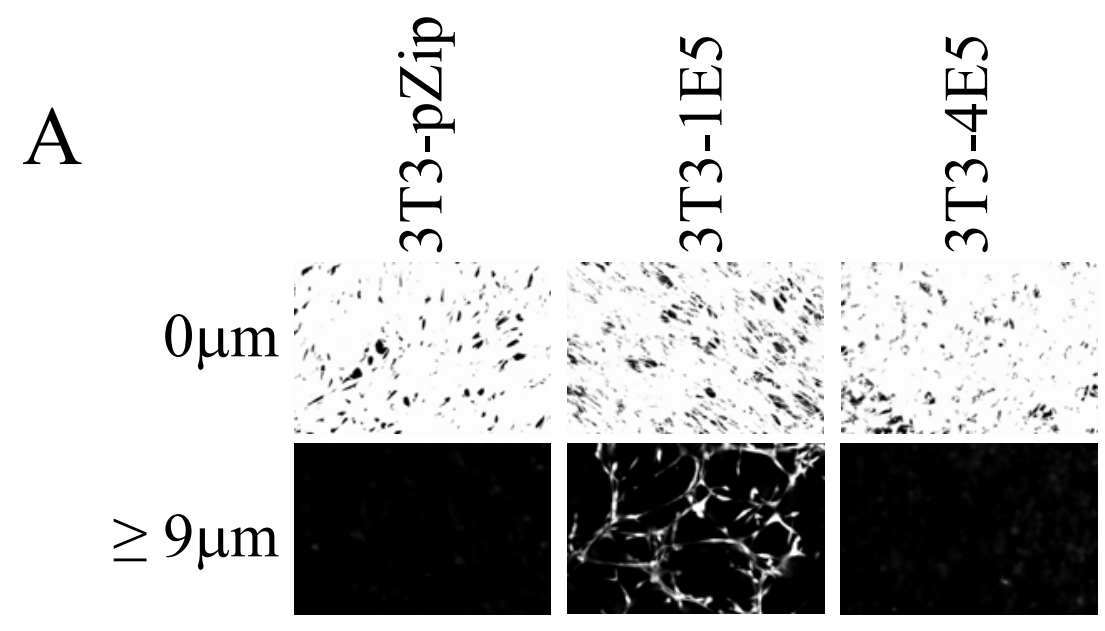

B

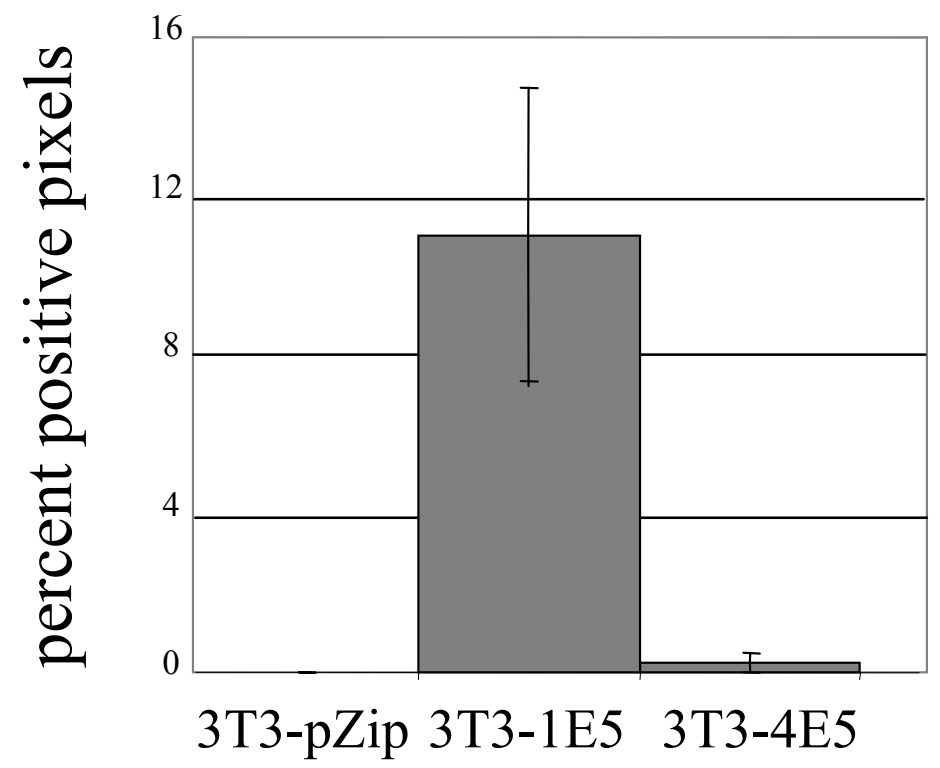

Tsirimonaki et al., Figure 5 


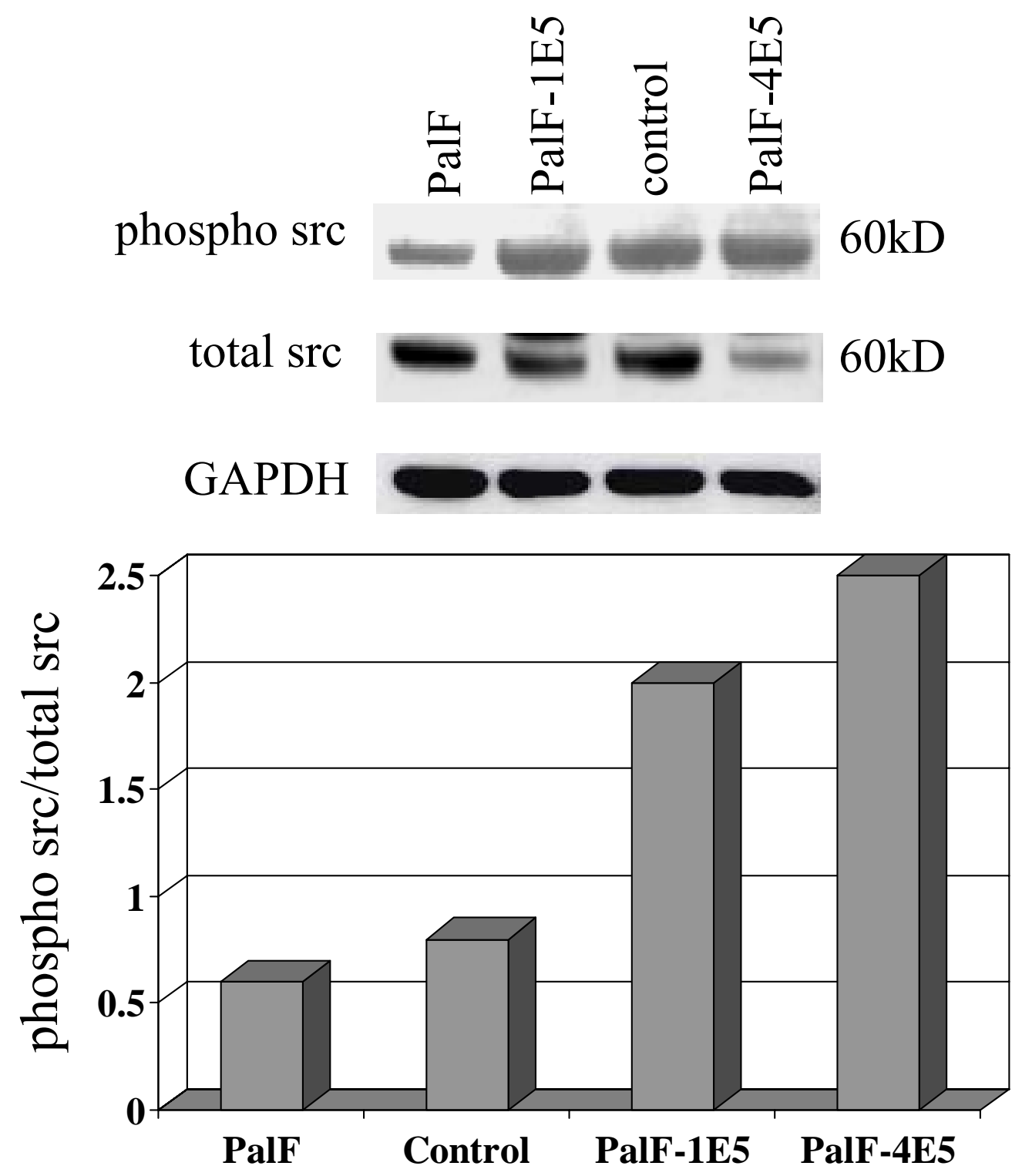

Tsirimonaki et al., Figure 6A 

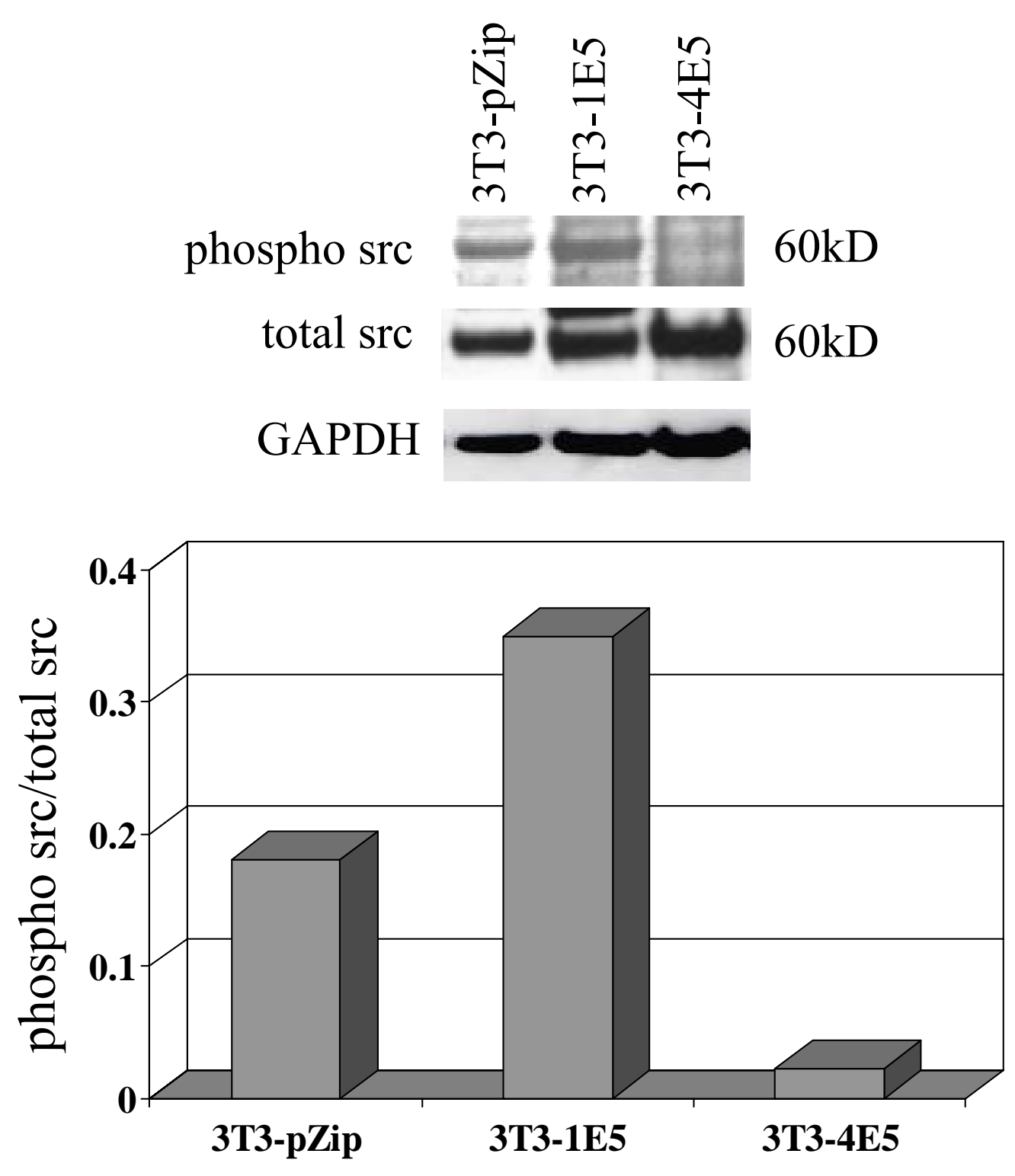

Tsirimonaki et al., Figure 6B 